\title{
Proton radiotherapy for pediatric tumors: review of first clinical results
}

\author{
Barbara Rombi ${ }^{{ }^{*}}$, Sabina Vennarini ${ }^{1 \dagger}$, Lorenzo Vinante ${ }^{1,2+}$, Daniele Ravanelli ${ }^{1}$ and Maurizio Amichetti ${ }^{1}$
}

\begin{abstract}
Radiation therapy is a part of multidisciplinary management of several childhood cancers. Proton therapy is a new method of irradiation, which uses protons instead of photons. Proton radiation has been used safely and effectively for medulloblastoma, primitive neuro-ectodermal tumors, craniopharyngioma, ependymoma, germ cell intracranial tumors, low-grade glioma, retinoblastoma, rhabdomyosarcoma and other soft tissue sarcomas, Ewing's sarcoma and other bone sarcomas. Moreover, other possible applications are emerging, in particular for lymphoma and neuroblastoma. Although both photon and proton techniques allow similar target volume coverage, the main advantage of proton radiation therapy is to sparing of intermediate-to-low-dose to healthy tissues. This characteristic could translate into clinical reduction of side effects, including a lower risk for secondary cancers. The following review presents the state of the art of proton therapy in the treatment of pediatric malignancies.
\end{abstract}

Keywords: Proton radiotherapy, Pediatric tumors, Late effects, Secondary tumors

\section{Review}

\section{Introduction}

Recent therapy progress has improved life expectancy in pediatric cancer patients, with a 5-year overall survival (OS) that increased from 39\% in 1960 higher than $80 \%$ in 2004 [1]. Radiotherapy (RT) is a fundamental part of the multimodality treatment applied to achieve local (LC) and regional control in solid malignancies. Unfortunately, as survivors live longer, they are at risk of experiencing late effects from their treatments, including radiation. For this reason, several combined approaches with chemotherapy and surgery have been used to avoid or reduce RT. Despite of this, many children require radiation and remain at high risk for developing a multitude of serious long-term sequelae that result in psychological - social problems and reduce the quality of life of survivors [2].

Protontherapy (PT) is an external RT modality that uses protons instead of photons. These charged particles are accelerated by a cyclotron or synchrotron to reach high energies. When a proton beam enters the body, it delivers a constant dose all through the end of the range where all the remaining energy is deposited within a few

\footnotetext{
*Correspondence: barbara.rombi@apss.tn.it

${ }^{\dagger}$ Equal contributors

${ }^{1}$ Unità Operativa di Protonterapia, Azienda Provinciale per i Servizi Sanitari (APSS), Trento, Italy

Full list of author information is available at the end of the article
}

millimeters (Bragg peak). The energy and the intensity of the beam can be varied to obtain a longitudinal translation (SOBP: spread-out Bragg peak) and cover the entire target volume. In comparison to high-energy photon treatment, the potential advantages of protons include distal dose fall off, without dose beyond the end of the range, reduction of the dose proximal to the target and reduction of the integral dose (Figure 1). Biologically, protons have not demonstrated a significant advantage over photons, with a relative biological effectiveness of protons of 1.1 compared to photons (1Gy radiobiological equivalent $(\mathrm{RBE})=1 \mathrm{~Gy} \times 1.1)$ [3]. Therefore, tumor control is predicted to be the same for both techniques, but the physical properties of protons allow a better sparing of normal tissues, with the consequent reduction of acute and late toxicities. This ability is particularly important during pediatric age, when the growing organs are very sensitive to radiation effects.

The long life expectancy of survivors and the presence of genetic mutations that induce malignancies (e.g. the $\mathrm{RB}$ gene mutation in retinoblastoma) are two factors that cause a significant risk for secondary cancers. The incidence of radiation-induced tumors expected to be reduced using PT, but longer follow-ups are needed for a clinical evidence [4-6]. 


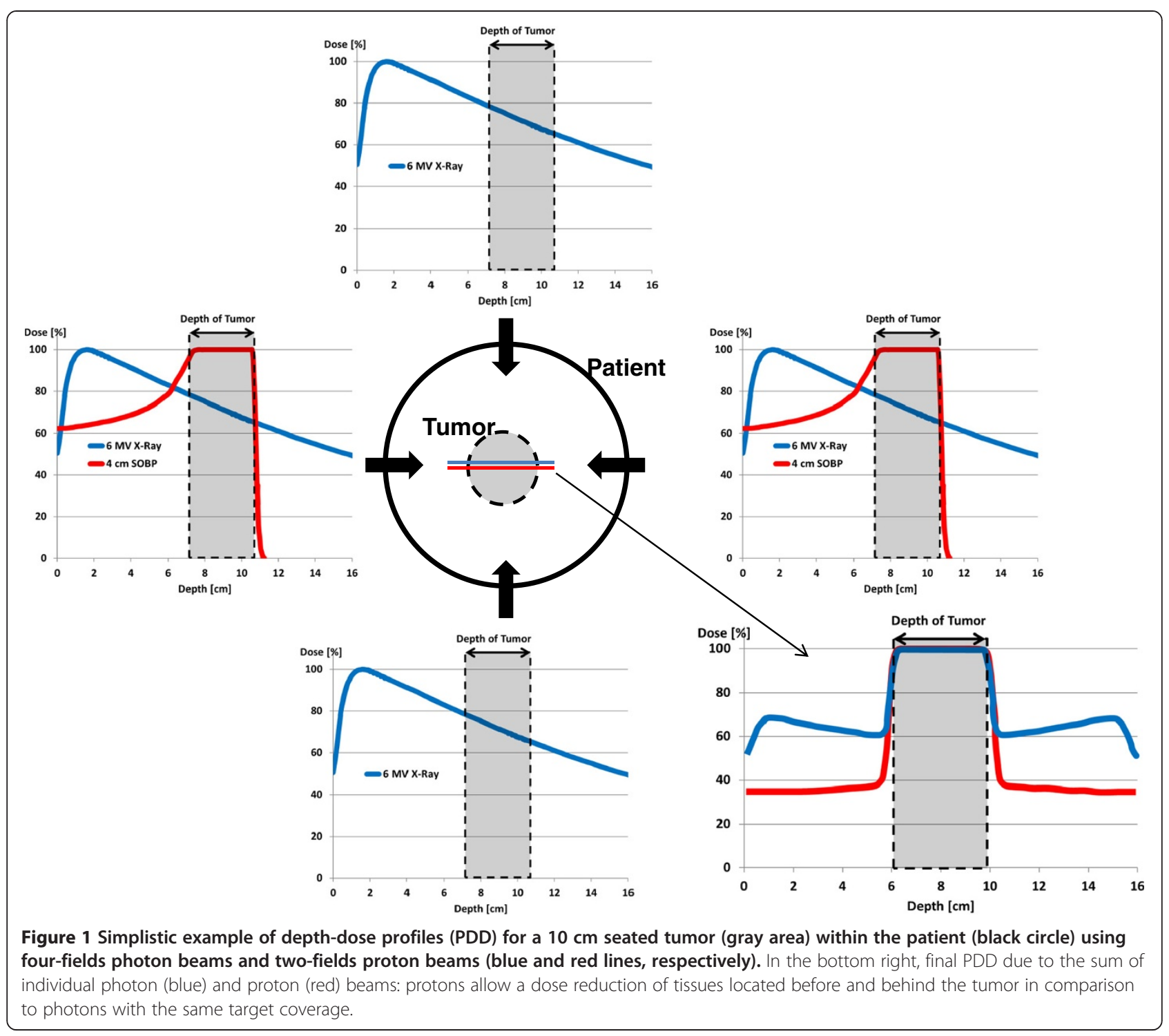

In a recent review [7], Merchant pointed out some controversial aspects regarding the clinical use of proton therapy for pediatric tumors: the limited number of proton centers which are not geographically well-distributed in the United States country, the lack of proton treatment availability in a timely way, the radiobiological uncertainties of protons might mean substantial differences in dose-volume histogram for proton and photon and that require a better understanding of the radiobiological differences between the two modalities. He concluded that the real benefit of proton over photon therapy based on toxicity reduction would only be realized once proton therapy data will be available for comparison with best existing photon outcome data.
For a definitive spread of this radiation modality, the benefits of protons should be evaluated by results from clinical trials. To date, nearly all-exiting studies on Children's Oncology Group (COG) for pediatric sarcoma including RMS, CNS tumors, Neuroblastoma, allow proton therapy as radiation treatment modality. In other countries, PT is not always a recognized as integral component of clinical trials. Up to now, although the clinical use of protons has grown, the proportion of patients treated by protons remains still small. One of the reasons is that many proton accelerators were not hospital-based, and were unable to accommodate children that required daily anesthesia and a proton treatment in a timely manner. Another aspect is that the allocation of proton treatment 
slots in US depends mostly on insurance status. The patients who have proton treatments reimbursed by insurance are significantly smaller than the population who should receive PBT [8].

There are also some physical aspects to consider: as proton technology is still evolving, PT facilities face many issues regarding the treatment planning and targeting of protons. Some of them are: to predict dose deposition due to range uncertainties by modern treatment planning system, the impact of anatomical changing tissue density during proton treatment, breathing, uncertainty regarding RBE [9]. Concern has also been raised about introducing this expensive technology into medical practice and the potential impact on the cost of health care. It is worth noting that while the initial cost of treatment with protons is higher than that of photon therapy, reduced side effects resulting an overall cost savings over a lifetime.

Currently, PT is used in the treatment of intracranial tumors such as ependymoma, medulloblastoma, primitive neuro-ectodermal tumors (PNET), craniopharyngioma, germinoma, and low-grade glioma. Regarding extracranial malignancies, PT is used in case of retinoblastoma, soft-tissue and bone sarcomas of head and neck and paraspinal or pelvic regions. The first clinical results of PT in pediatric oncology are described in the next section.

\section{First clinical results Ependymoma}

The current therapeutic approach for localized ependymoma is the maximal tumor resection followed by RT. A dosimetric comparison study [10] between PT and intensity modulated photon radiation therapy (IMRT) showed a significant dose reduction from radiation of cochlea, temporal lobes, whole brain and hypothalamus with PT, while target coverage and dose to organs close to the target (e.g. brainstem) were similar. An additional sparing of healthy tissues would be possibly obtainable with intensity modulated PT (IMPT).

Recently, MacDonald et al. [11] reported the outcome of 70 patients with localized ependymoma (73\% infratentorial, 27\% supratentorial) after total or subtotal resection followed by PT. At a median follow-up of 46 months, 3-year LC, progression-free survival (PFS) and OS were $83 \%, 76 \%$ and $95 \%$, respectively. Subtotal resection was significantly associated with worse PFS (54\% vs. $88 \%$, $\mathrm{p}=0.001)$ and OS $(90 \%$ vs. $97 \%, \mathrm{p}=0.001)$. Only three patients had pituitary gland deficiency after PT (growth hormone deficiency and hypothyroidism) and two patients had hearing loss. Regarding potential neurocognitive sequelae, mean intelligence and adaptive skills after PT were stable in comparison to the baseline evaluation and did not changed in different age groups. No cases of secondary malignancies were identified.
Amsbaugh et al. [12] published the clinical results of 8 cases of spinal ependymoma that received PT after local recurrence ( 3 cases) or as part of their primary treatment (5 cases). The mean prescribed dose was $51.1 \mathrm{~Gy}$ (RBE). After a mean follow-up of 26 months LC, PFS and OS were $100 \%$. There were no events of high-grade toxicity. In comparison to photon therapy, the dose to the Organs At Risk (OARs) anterior to the vertebral bodies (i.e. thyroid, heart, small bowel, stomach) was dramatically reduced.

In conclusion, both dosimetric studies and the first clinical results suggest that treating ependymoma with PT can better preserve hearing, neuro-endocrine and neurocognitive functioning, with similar rates of tumor control obtained by photon RT.

\section{Craniopharyngioma}

RT is usually delivered after limited surgery, at the time of first diagnosis or at progression, or in exclusive RT setting [13]. The combined approach with limited surgery and irradiation reduces the late toxicity of OARs placed in the chiasmatic or pre-chiasmatic region (i.e. optic chiasm, hippocampi, hypothalamus and pituitary gland) in comparison to radical surgery alone [14]. Merchant et al. [15] evaluated 3 dimensional imaging and treatment-planning data including targeted tumor and normal tissues volumes (entire brain, temporal lobes, cochleas, hypothalamus) of 10 craniopharyngioma patients. Dose-volume data were compared based on proton and photon treatment modality using dose-cognitive effects models. Craniopharyngioma target volume coverage was similar for both treatment modalities. The differences between proton and photon dosimetry showed an advantageous sparing of cochleae, hypothalamus, and normal tissue volumes such as supratentorial brain or temporal lobes, which received less of the low and intermediate doses. Those differences when applied to longitudinal models of radiation dose-cognitive effects resulted in higher IQ scores for craniopharyngioma patients. Figure 2 shows an example of dosimetric comparison between PT and IMRT plans for craniopharyngioma case.

Boehling et al. [16] compared three-dimensional conformal PT (3D-CPT), IMPT and IMRT plans of 10 pediatric patients affected by craniopharyngioma. The target volume coverage was adequate for all modalities but 3D-CPT and even more IMPT reduced the integral dose to hippocampus, dentate gyrus, subventricular zone, brain tissue, cerebellum, brainstem and major cerebral arteries. Similar results were obtained by Beltran et al. [17] who compared the same techniques in 14 craniopharyngioma cases. The best conformal technique was IMPT, which has high sensitivity to target volume changes.

Preliminary clinical results of 16 craniopharyngioma patients treated with post-operative PT to a total dose of 


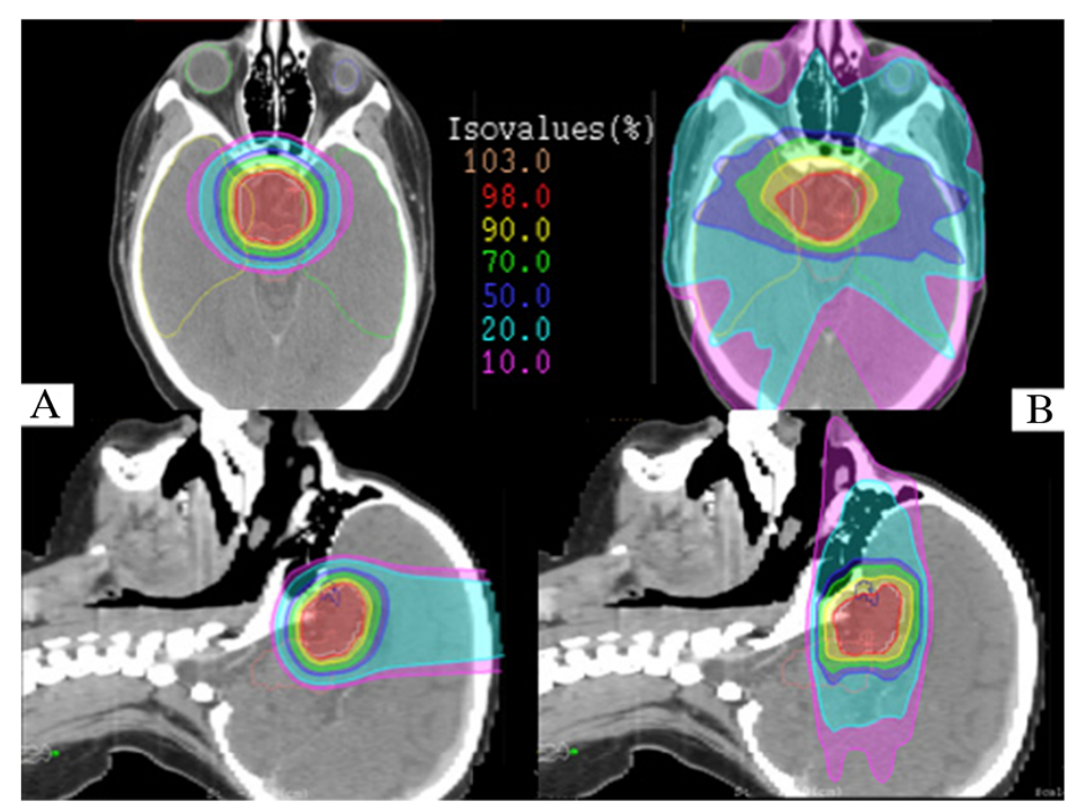

Figure 2 Axial and sagittal isodose distributions comparing intensity-modulated proton therapy (IMPT) (A) and intensity-modulated radiotherapy (IMRT) (B). For IMPT plan 3 beam angles were used (1 vertex, 2 symmetric lateral off-axis vertex). For IMRT plan 7 equidistant and coplanar beams were used. PTV (white), right temporal lobe (yellow), left temporal lobe (green), brainstem (pink), left eye (light blue), right eye (light green), chiasma (blue) are outlined. The IMPT plan improved sparing of the temporal lobes, orbital structures and both optic nerves. The integral dose to the brain tissue is decreased with IMPT.

50.4 - 59.4 Gy(RBE) were reported by Luu et al. [18]. After a mean follow-up of 62 months, LC and OS were $93 \%$ and $80 \%$, respectively, while $75 \%$ of patients were free from late toxicity. The effect of combined surgery and PT on quality of life was investigated by Laffond et al. [19] with specific questionnaires administered to 23 families. Depression was registered in $48 \%$ of cases, $38 \%$ had dysexecutive symptoms and the majority of families felt "very concerned" by the disease.

In summary, PT showed to be indicated as part of a multidisciplinary management with the goal to reduce late sequelae, especially in regards to neurocognitive functioning.

\section{Medulloblastoma and primitive neuro-ectodermal tumor (PNET)}

The standard therapy for children older than 3 years with medulloblastoma and PNET is maximal safe resection followed by craniospinal irradiation (CSI) in addition to posterior fossa or tumor bed boost and chemotherapy. Patients younger than 3 years, the role of CSI is still controversial due to important side effects. Using photon beams, the exit dose from the spinal field results in irradiation of normal tissues anterior to the spine i.e. thyroid, heart, lungs, bowel, ovaries, breast and uterus. PT is better able to spare these organs because of the distal dose fall-off in the few millimeters anterior to the vertebral bodies.

Zhang et al. [20] evaluated the predicted risks of cardiac toxicities for a 4-year-old boy receiving photon or proton irradiation CSI for medulloblastoma. They calculated the relative risk (RR) values using a linear risk model and the normal tissue complication probability (NTCP) values using Lyman model. The RR values of cardiac toxicity were 1.28 with protons versus 8.39 with photons. The predicted ratios of NTCP (photons/protons) were much less than unit. They concluded that PT CSI carried a lower risk of radiogenic cardiac toxicity compared to photon CSI.

The relative risk of premature ovarian failure (RRPOF) after cranio-spinal proton radiotherapy was investigated by Pérez-Andújar and colleagues [21]. They calculated the equivalent dose delivered to the ovaries of an 11year old girl from therapeutic and stray radiation and then predicted the percentage of ovarian primordial follicles killed by radiation and used this as a measure of RRPOF among the three radiotherapies. Proton radiotherapy had a lower RRPOF than conventional photon radiotherapy or IMRT for all sets of ovaries, regardless of uncertainties in ovarian location.

Clair et al. [22] generated three plans of a patient affected by medulloblastoma, while comparing conventional $\mathrm{x}$-rays, IMRT and PT. PT resulted to be the best technique to 
reduce the dose to structures located beyond the vertebral body and to the cochlea, pituitary, hypothalamus, temporomandibular joint, parotid and pharynx. The dosimetric advantage of protons over photons RT was documented by Lin et al. [23] in 9 patients who underwent posterior fossa irradiation. They reported better sparing of cochlea and temporal lobe by PT.

Moeller et al. [24] performed pre- and one-year post-PT audiometric testing in patients treated for medulloblastoma who also received platinum-based chemotherapy. The hearing function of 35 ears was analyzed: the rate of high-grade ototoxicity was low (5\%) and the sensitivity to low frequencies was preserved. Jimenez et al. [25] reported the outcomes of 15 very young children (age $<5$ years) treated with upfront chemotherapy and subsequent PT (local volume for all patients, with the addition of CSI in 11) for medulloblastoma and PNET tumors. At a median follow-up of 39 months, one patient died for local failure and one from a non-disease related cause. The remaining 13 out of 15 were alive, free of recurrence. $13 \%$ patients developed grade 3 ototoxicity requiring hearing aids and $20 \%$ had grade 2 neuro-endocrinopathy. The IQ index remained stable during follow-up testing in comparison to baseline evaluation.

The potential reduction of secondary tumors is well documented by radiobiological predictive models [4-6], but clinical data from long term follow-ups are not yet available. This advantage could translate into new clinical indications, as for example the usefulness of breast cancer screening in survivors, considered mandatory after $\mathrm{x}$-ray therapy, that will be unnecessary after CSI with PT [26].

On the basis of dosimetric studies and early clinical outcome reports, PT should be strongly considered when logistically possible for tumors requiring CSI. A recent debate has risen in the radiation oncology community $[27,28]$ about PT as being the only ethically appropriate radiation treatment for medulloblastoma. Hopefully, longterm follow-up will give the answer.

\section{Germ cell tumor}

RT has a fundamental role for these malignancies that are divided into two histological categories: germinomas and non-germinomatous germ cells tumors (NGGCT). For diffuse germinomas and NGGCT RT is delivered as CSI plus a boost to the initial tumor. For localized germinomas, the target volume is limited to the whole ventricular system followed by a boost to the residual disease [29]. MacDonald et al. [30] compared 3D-CPT and IMPT plans of 22 germinoma patients with IMRT obtaining similar target coverage with all three techniques but a substantial sparing of brain tissue with PT. LC and OS were $100 \%$ after a median follow-up of 28 months. The treatment was well tolerated in terms of acute toxicity. Only 4 patients developed late toxicity ( 2 hypothyroidism and 2 growth hormone deficiency), while no neurocognitive or auditory toxicity were detected. This is currently the only study that shows the early outcome of patients with germ cell tumors treated with PT.

Although preliminary, the results are interesting from both the clinical and the dosimetrical point of view not only for the acute toxicity reduction but also for the potential advantage on late side effects, such as neurocognitive sequelae due to the lower irradiation of the temporal lobes.

\section{Low-grade glioma}

Surgical resection is the main treatment in low-grade glioma. RT is used particularly for deeply placed tumors, after incomplete surgery or in the definitive setting after post-chemotherapy progression [31]. Hug et al. [32] published the results of 27 patients treated with PT at doses of 50.4-63 Gy(RBE) after a median follow-up of 3.3 years. PT was used as an adjuvant $(44 \%)$ or salvage (56\%) intent obtaining LC and OS rate of $87 \%$ and $93 \%$ for diencephalic tumors, $71 \%$ and $86 \%$ for hemispheric tumors, $60 \%$ and $60 \%$ for tumors placed in the brainstem. All patients with stable disease maintained a good performance status.

In gliomas of the optic nerves, RT is a treatment option that should be considered in case of local progression after surgery or for unresectable tumors. Fuss et al. [33] compared PT plans with 3D-CRT and lateral photon beam techniques and showed similar conformity and homogeneity of dose coverage of the target, but a better sparing of frontal and temporal lobes, contralateral optic nerve and pituitary gland.

RT in low-grade gliomas is associated with possible negative effects in neurocognitive, vascular, visual and endocrine functioning. PT has shown promising results in reducing these adverse events thanks to a better sparing of healthy tissues (i.e. useful vision has been maintained in $57 \%$ of patients and improved in $29 \%$ as reported by Fuss et al. [33]) while similar local control was obtained.

\section{Soft tissue Sarcoma (STS)}

RT is usually part of the multimodal approach of STS together with chemotherapy and surgery. PT is usually employed to reduce side effects in cases where the tumor is in close proximity to critical structures. Timmermann et al. [34] treated 16 sarcomas located in different sites, with a total dose of 50.4 - 61.2 Gy(RBE). After a median follow-up of 18.6 months, two-year PFS and OS were $71.6 \%$ and $69.3 \%$, respectively; four patients had local failure. PT was well tolerated. 


\section{Rhabdomyosarcoma (RMS)}

Yock et al. [35] compared 3D photon and proton plans of seven patients with orbital RMS treated with PT and evaluated the rate of clinical late effects. PT showed dosimetric advantage limiting the dose to the brain, pituitary grand, hypothalamus, temporal lobes and ipsi/ contralateral orbital structures. After a median follow up of 6.3 years, only one local recurrence had occurred. Late toxicity was limited, excellent vision was maintained. Two patients requiring eye drops and none of patients developed neuroendocrine dysfunction. All patients had facial dimorphism, 29\% mild bone asymmetry and $71 \%$ enophthalmos. Kozak et al. [36] compared PT and IMRT plans of 10 parameningeal RMS patients. PT reduced dose to orbital structures, optic pathways, whole brain temporal lobes, brainstem, pituitary, hypothalamus, parotid and lacrimal glands with similar target volume coverage.

Childs et al. [37] published the clinical outcome of 17 patients with parameningeal RMS treated with PT to a dose ranged between 50.4 and 56 Gy (RBE). With a median follow-up of 50 months, the 5-year PFS and 5-year PFS were $59 \%$ and $64 \%$, respectively, comparable with historical photon cohorts. Late effects included failure to maintain height velocity $(n=3)$, endocrine deficits $(n=2)$, mild facial hypoplasia $(n=7)$, failure of tooth eruption $(\mathrm{n}=3)$, dental caries $(\mathrm{n}=5)$ and chronic nasal/sinus congestion $(n=2)$. Functional results were better in comparison to those observed in photon studies $[38,39]$.

Cotter et al. [40] compared IMRT and PT plans for 7 patients with bladder/prostate RMS. PT led to a significant decrease in mean dose to bladder, testes, femoral heads, growth plates and pelvic bones compared to IMRT. After a median follow-up of 27 months, two recurrences were observed. Late effects related to PT were very limited: only one patient had intermittent hematuria.

\section{Ewing sarcoma}

In the multimodality management of Ewing sarcoma, RT is usually used in post-operative setting for patients with close or positive resection margins after poor response post-chemo or as a definitive intent for unresectable tumors. Rombi et al. [41] published initial clinical outcome of 30 Ewing's sarcoma patients treated with PT at a median prescribed dose of $54 \mathrm{~Gy}(\mathrm{RBE})$. At a median follow-up of 38.4 months, 3-year LC, PFS, and OS were $86 \%, 60 \%$, and $89 \%$, respectively. The PT was very well tolerated and acute Grade 3 side effects occurred in 6 cases ( 5 skin desquamation and 1 fatigue). Five patients (16.7\%) who received surgical laminectomy prior to radiation developed scoliosis/kyphosis (three mild, one moderate, one severe) and $20 \%$ had permanent skin changes. Four patients developed secondary hematological tumors (three acute myeloid leukemia and one myelodysplastic syndrome), potentially due to high doses of alkylating antineoplastic agents.

In conclusion, PT may be indicated in selected cases of STS, RMS and Ewing sarcoma, especially in orbital, parameningeal, paraspinal and pelvic sites to mitigate late toxicity. Further investigations to evaluate the impact of PT on bone impairment are needed.

\section{Chordoma and Chondrosarcoma}

In chordoma and chondrosarcoma RT may be prescribed in post-operative setting or with exclusive intent. It is usually delivered at high doses up to 70-76 Gy(RBE). These tumors are always in close proximity to critical structures, as brainstem, optic pathways, brain tissue and spinal cord. Chordomas and chondrosarcomas are historically the typical pathologies referred to PT facilities. Habrand et al. [42] evaluated clinical results of 30 children treated with combined photon and proton RT, with a mean follow up of 26.5 months. Five year PFS was $100 \%$ for chondrosarcoma and $77 \%$ for chordoma and 5-year OS was 100\% for chondrosarcoma and $81 \%$ for chordoma. The acute tolerance to PT treatment was very good; only one late severe toxicity case (hearing loss) was registered. Rombi et al. [43] reported the outcome of 26 patients treated with PT after a mean follow up of 46 months. In chordoma cohort, the 5-year LC and 5-year OS were $81 \%$ and $89 \%$, respectively; in chondrosarcoma cohort, the 5-year LC and 5 -year OS were $80 \%$ and $75 \%$, respectively. Relatively few $(19 \%)$ late complications were described, with no events of severe grade.

PT is considered a useful RT modality for chordoma and chondrosarcoma due to the capability to escalate the dose with acceptable rates of late toxicity.

\section{Retinoblastoma}

$\mathrm{RT}$ is commonly used to preserve the eye and the visual function in advanced retinoblastomas or in the postoperative setting for high-risk tumors. Krengli et al. [44] showed that PT is useful to reduce the integral dose to healthy tissue, decreasing the risk of bone impairment and functional sequelae. Also, secondary malignancies could be reduced particularly in children with hereditary $\mathrm{RB}$ gene mutation, associated with a higher risk of radiationinduced tumors. Sethi et al. [45] have recently compared the risk for secondary malignancies in patients with retinoblastoma who were treated with PT (55 patients) and photon radiotherapy (31 patients). The median follow up was 6.9 years in proton cohort and 13.1 years in photon cohort. Ten-year cumulative incidence of radiationinduced second malignancies was $0 \%$ vs.14\% $(\mathrm{p}=0.015)$, respectively.

PT Department at Massachusetts General Hospital of Boston is treating patients with advanced or high-risk 
retinoblastoma with preliminary good clinical results in regards to secondary-tumor reduction, functional and cosmetic outcomes [45].

\section{Neuroblastoma}

RT is currently part of treatment for high-risk neuroblastoma and it is usually delivered on primary tumor site with limited doses after neoadjuvant chemotherapy and surgery. The role of PT in neuroblastoma is mainly investigated for the retroperitoneal site, which accounts for about the $40 \%$ of all cases. Hattangadi et al. [46] compared dosimetrically 3 different plans (IMRT, 3D$\mathrm{CPT}$, and IMPT) of 9 patients. The dosimetric advantage of 3D-CPT over IMRT was demonstrated in all cases, while IMPT allowed additional sparing of kidneys, lungs and heart. In this paper, all cases were treated with 3D-CPT and at a median follow up of 38 months, and no local failures were detected. Few acute and late side effects were detected without high-grade toxicity.

Hill Kayser et al. [47] compared 3D-CPT with IMRT of 13 patients in order to choose the most appropriate technique in relation to tumor location. For 9 patients with lateralized disease, PT offered sparing of the contralateral kidney but in two cases, IMRT improved overall bilateral renal sparing. They concluded that PT offered optimal combination between target coverage and OARs sparing (i.e. bowel, both kidneys, liver, heart, lungs) in most of the cases (11/13) with $100 \%$ of local control occurred after a mean follow up of 16 months.

Fuji et at. [48] compared 3D-CPT, 3D-CRT and IMRT plans of 5 cases and obtained similar results regarding dose sparing. Additionally, by using 3D-CPT the secondary cancer risk calculated using a model, which took in account the rates of cell killing, repopulation and neutron dose from treatment, was lower in all organs evaluated (i.e. liver, stomach, small intestine, colon, bone) except in the pancreas.

The dosimetric evaluations and the first clinical results of PT in high-risk neuroblastoma are promising. The potentiality of PT to reduce the dose to organs outside the target volume could improve the tolerance of a very aggressive multimodal treatment and decrease the incidence of secondary cancer.

\section{Lymphoma}

In recent years, numerous comparative studies have been conducted to evaluate the potential role of PT in mediastinal lymphoma. Hoppe et al. [49] evaluated the plans of stage II-III Hodgkin's lymphoma (HL) cases with mediastinal location and they confirmed the advantage of 3D-CPT over conformal RT and IMRT to reduce the dose to the heart, lungs, esophagus, thyroid and breast. The same authors [50] published a second article mainly focused on heart structures, in particular the coronary arteries, with the potential reduction of late ischemic damage by PT use. Andolino et al. [51] found that using $3 \mathrm{D}-\mathrm{CPT}$ in pediatric female patients with stage II-HL the dose to the breast was reduced of $80 \%$.

In dosimetric comparison studies of protons and photons in several tumor types and locations, PT appears promising in reducing the dose to OARs; it could translate into reduced incidence of secondary cancer (especially breast cancer) but clinical evidence are still limited.

\section{Conclusions}

RT is effective in increasing local control in several pediatric tumors, but it is often associated with severe late effects, including secondary tumors. The physical advantages of protons, which decrease the dose to healthy tissues, are promising in achieving significant clinical benefits. Dosimetric comparison studies pointed out the superiority of protons over photons in several tumor locations. Clinical data are still limited, but the first evidence generally confirmed an outcome similar to historic photon cohort, but better tolerance to PT and few side effects, that could have positive impact also on the survivors' quality of life.

Several proton facilities have started their activity during the last decade and new centers are planned worldwide. The spread of the PT centers in the near future will make this technology easily accessible to pediatric patients. With the increase of follow-up time, the evidence in favor of PT could become more solid. Specialized teams of radiation oncologists, medical physicists, anesthesiologists, psychologists and nurses are needed and new observational protocols should be designed to investigate the benefits on late toxicity and quality of life.

\section{Abbreviations}

RT: Radiotherapy; PT: Protontherapy; IMRT: Intensity modulated radiotherapy; IMPT: Intensity modulated protontherapy; 3D-CPT: Three dimensional conformal proton therapy; 3D-CRT: Three dimensional conformal radiotherapy; RBE: Radiobiological equivalent; OAR: Organ at risk; OS: Overall survival; LC: Local control; PFS: Progression-free survival; CSI: Craniospinal irradiation; PNET: Primitive neuro-ectodermal tumors; NGGCT: Non-germinomatous germ cells tumors; HL: Hodgkin's lymphoma.

\section{Competing interests}

The authors declare that they have no competing interests.

\section{Authors' contributions}

SV, LV and BR wrote the manuscript, DR prepared the figures, BR and MA critically reviewed the article. All authors read and approved the final manuscript.

\section{Acknowledgements}

The authors would like to thank Dr. Valentina Piffer for her English revision of the text.

\section{Author details}

${ }^{1}$ Unità Operativa di Protonterapia, Azienda Provinciale per i Servizi Sanitari (APSS), Trento, Italy. ${ }^{2}$ Dipartimento di Medicina, Università di Padova, Padova, Italy. 
Received: 26 March 2014 Accepted: 4 August 2014

Published online: 26 September 2014

\section{References}

1. Robison LL, Armstrong GT, Boice JD, Chow EJ, Davies SM, Donaldson SS, Green DM, Hammond S, Meadows AT, Mertens AC, Mulvihill JJ, Nathan PC, Neglia JP, Packer RJ, Rajaraman P, Sklar CA, Stovall M, Strong LC, Yasui Y, Zeltzer LK: The childhood cancer survivor study: a National Cancer Institute-supported resource for outcome and intervention research. J Clin Oncol 2009, 27(14):2308-2318.

2. Ishida Y, Sakamoto N, Kamibeppu K, Kakee N, Iwai T, Ozono S, Maeda N, Okamura J, Asami K, Inada H, Honda M, Horibe K: Late effects and quality of life of childhood cancer survivors: part 2: impact of radiotherapy. Int J Hematol 2010, 92(1):95-104.

3. Paganetti H, Niemierko A, Ancukiewicz M, Gerweck LE, Goitein M, Loeffler JS, Suit HD: Relative biological effectiveness (RBE) values for proton beam therapy. Int J Radiat Oncol Biol Phys 2002, 53(2):407-421.

4. Miralbell R, Lomax A, Cella L, Schneider U: Potential reduction of the incidence of radiation-induced second cancers by using proton beams in the treatment of pediatric tumors. Int J Radiat Oncol Biol Phys 2002, 54(3):824-829

5. Newhauser WD, Fontenot JD, Mahajan A, Kornguth D, Stovall M, Zheng Y, Taddei PJ, Mirkovic D, Mohan R, Cox JD, Woo S: The risk of developing a second cancer after receiving craniospinal proton irradiation. Phys Med Biol 2009, 54(8):2277-2291.

6. Brodin NP, Munck AF, Rosenschöld P, Aznar MC, Kiil-Berthelsen A, Vogelius IR, Nilsson P, Lannering B, Björk-Eriksson T: Radiobiological risk estimates of adverse events and secondary cancer for proton and photon radiation therapy of pediatric medulloblastoma. Acta Oncol 2011, 50(6):806-816.

7. Merchant TE: Clinical controversies: proton therapy for pediatric tumors. Semin Radiat Oncol 2013, 23(2):97-108.

8. Bekelman JE, Asch DA, Tochner Z, Friedberg J, Vaughn DJ, Rash E, Raksowski K, Hahn SM: Principles and reality of proton therapy treatment allocation. Int J Radiat Oncol Biol Phys 2014, 89(3):499-508.

9. Engelsman M, Schwarz M, Dong L: Physics controversies in proton therapy. Semin Radiat Oncol 2013, 23(8):88-96.

10. MacDonald SM, Safai S, Trofimov A, Wolfgang J, Fullerton B, Yeap BY, Bortfeld T, Tarbell NJ, Yock T: Proton radiotherapy for childhood ependymoma: initial clinical outcomes and dose comparisons. Int J Radiat Oncol Biol Phys 2008, 71(4):979-986.

11. Macdonald SM, Sethi R, Lavally B, Yeap BY, Marcus KJ, Caruso P, Pulsifer M, Huang M, Ebb D, Tarbell NJ, Yock TI: Proton radiotherapy for pediatric central nervous system ependymoma: clinical outcomes for 70 patients. Neuro Oncol 2013, 15(11):1552-1559.

12. Amsbaugh MJ, Grosshans DR, McAleer MF, Zhu R, Wages C, Crawford CN, Palmer M, De Gracia B, Woo S, Mahajan A: Proton therapy for spinal ependymomas: planning, acute toxicities and preliminary outcomes. Int $\mathrm{J}$ Radiat Oncol Biol Phys 2012, 83(5):1419-1424.

13. Manaka S, Teramoto A, Takakura K: The efficacy of radiotherapy for craniopharyngioma. J Neurosurg 1985, 62(5):648-656.

14. Fischer EG, Welch K, Shillito J, Winston KR, Tarbell NJ: Craniopharyngioma in children: long-term effects of conservative surgical procedures combined with radiation therapy. J Neurosurg 1990, 73(4):534-540.

15. Merchant TE, Hua CH, Shukla $H$, Ying $X$, Nill S, Oelfke U: Proton versus photon radiotherapy for common pediatric brain tumors: comparison of models of dose characteristics and their relationship to cognitive function. Pediatr Blood Cancer 2008, 51(1):110-117.

16. Boehling NS, Grosshans DR, Bluett JB, Palmer MT, Song X, Amos RA, Sahoo N, Meyer JJ, Mahajan A, Woo SY: Dosimetric comparison of three-dimensional conformal proton radiotherapy, intensity-modulated proton therapy, and intensity-modulated radiotherapy for treatment of pediatric craniopharyngiomas. Int J Radiat Oncol Biol Phys 2012, 82(2):643-652.

17. Beltran C, Roca M, Merchant TE: On the benefits and risks of proton therapy in pediatric craniopharyngioma. Int I Radiat Oncol Biol Phys 2012, 82(2):e281-e287.

18. Luu QT, Loredo LN, Archambeau JO, Yonemoto LT, Slater JM, Slater JD: Fractionated proton radiation treatment for pediatric craniopharyngioma: preliminary report. Cancer J 2006, 12(2):155-159.

19. Laffond C, Dellatolas G, Alapetite C, Puget S, Grill J, Habrand JL, Doz F, Chevignard M: Quality-of-life, mood and executive functioning after childhood craniopharyngioma treated with surgery and proton beam therapy. Brain Inj 2012, 26(3):270-281.

20. Zhang R, Howell RM, Homann K, Giebeler A, Taddei PJ, Mahajan A, Newhauser WD: Predicted risks of radiogenic cardiac toxicity in two pediatric patients undergoing photon or proton radiotherapy. Radiat Oncol 2013, 8(1):184.

21. Pérez-Andújar A, Newhauser WD, Taddei PJ, Mahajan A, Howell RM: The predicted relative risk of premature ovarian failure for three radiotherapy modalities in a girl receiving craniospinal irradiation. Phys Med Biol 2013, 58(10):3107-3123.

22. St Clair WH, Adams JA, Bues M, Fullerton BC, La Shell S, Kooy HM, Loeffler JS, Tarbell NJ: Advantage of protons compared to conventional X-ray or IMRT in the treatment of a pediatric patient with medulloblastoma. Int J Radiat Oncol Biol Phys 2004, 58(3):727-734.

23. Lin R, Hug EB, Schaefer RA, Miller DW, Slater JM, Slater JD: Conformal proton radiation therapy of the posterior fossa: a study comparing protons with three-dimensional planned photons in limiting dose to auditory structures. Int J Radiat Oncol Biol Phys 2000, 48(4):1219-1226.

24. Moeller BJ, Chintagumpala M, Philip JJ, Grosshans DR, MCAleer MF, Woo SY, Gidley PW, Vats TS, Mahajan A: Low early ototoxicity rates for pediatric medulloblastoma patients treated with proton radiotherapy. Radiat Oncol 2011, 6:58.

25. Jimenez RB, Sethi R, Depauw N, Pulsifer MB, Adams J, McBride SM, Ebb D, Fullerton BC, Tarbell NJ, Yock TI, Macdonald SM: Proton radiation therapy for pediatric medulloblastoma and supratentorial primitive neuroectodermal tumors: outcomes for very young children treated with upfront chemotherapy. Int J Radiat Oncol Biol Phys 2013, 87(1):120-126.

26. Kumar RJ, Zhai H, Both S, Tochner Z, Lustig R, Hill-Kayser C: Breast cancer screening for childhood cancer survivors after craniospinal irradiation with protons versus $\mathrm{x}$-rays: a dosimetric analysis and review of the literature. J Pediatr Hematol Oncol 2013, 35(6):462-467.

27. Wolden SL: Protons for craniospinal radiation: are clinical data important? Int J Radiat Oncol Biol Phys 2013, 87(2):231-232.

28. Johnstone PA, McMullen KP, Buchsbaum JC, Douglas JG, Helft P: Pediatric CSI: are protons the only ethical approach? Int J Radiat Oncol Biol Phys 2013, 87(2):228-230.

29. Aoyama H, Shirato H, Ikeda J, Fujieda K, Miyasaka K, Sawamura Y: Induction chemotherapy followed by low-dose involved-field radiotherapy for intracranial germ cell tumors. J Clin Oncol 2002, 20(3):857-865.

30. MacDonald SM, Trofimov A, Safai S, Adams J, Fullerton B, Ebb D, Tarbell NJ, Yock TI: Proton radiotherapy for pediatric central nervous system germ cell tumors: early clinical outcomes. Int J Radiat Oncol Biol Phys 2011, 79(1):121-129.

31. Merchant TE, Kun LE, Wu S, Xiong X, Sanford RA, Boop FA: Phase II trial of conformal radiation therapy for pediatric low-grade glioma. $J$ Clin Oncol 2009, 27(22):3598-3604.

32. Hug EB, Muenter MW, Archambeau JO, DeVries A, Liwnicz B, Loredo LN, Grove Rl, Slater JD: Conformal proton radiation therapy for pediatric low-grade astrocytomas. Strahlenther Onkol 2002, 178(1):10-17.

33. Fuss M, Hug EB, Schaefer RA, Nevinny-Stickel M, Miller DW, Slater JM, Slater JD: Proton radiation therapy (PRT) for pediatric optic pathway gliomas: comparison with 3D planned conventional photons and a standard photon technique. Int J Radiat Oncol Biol Phys 1999, 45(5):1117-1126.

34. Timmermann B, Schuck A, Niggli F, Weiss M, Lomax AJ, Pedroni E, Coray A, Jermann M, Rutz HP, Goitein G: Spot-scanning proton therapy for malignant soft tissue tumors in childhood: first experiences at the Paul Scherrer Institute. Int J Radiat Oncol Biol Phys 2007, 67(2):497-504.

35. Yock T, Schneider R, Friedmann A, Adams J, Fullerton B, Tarbell N: Proton radiotherapy for orbital rhabdomyosarcoma: clinical outcome and a dosimetric comparison with photons. Int I Radiat Oncol Biol Phys 2005, 63(4):1161-1168.

36. Kozak KR, Adams J, Krejcarek SJ, Tarbell NJ, Yock TI: A dosimetric comparison of proton and intensity-modulated photon radiotherapy for pediatric parameningealrhabdomyosarcomas. Int I Radiat Oncol Biol Phys 2009, 74(1):179-186.

37. Childs SK, Kozak KR, Friedmann AM, Yeap BY, Adams J, MacDonald SM Liebsch NJ, Tarbell NJ, Yock TI: Proton radiotherapy for parameningeal rhabdomyosarcoma: clinical outcomes and late effects. Int I Radiat Oncol Biol Phys 2012, 82(2):635-642.

38. Raney RB, Asmar L, Vassilopoulou-Sellin R, Klein MJ, Donaldson SS, Green J, Heyn R, Wharam M, Glicksman AS, Gehan EA, Anderson J, Maurer HM: Late 
complications of therapy in 213 children with localized, nonorbital soft-tissue sarcoma of the head and neck: a descriptive report from the Intergroup Rhabdomyosarcoma Studies (IRS)-II and - III: IRS Group of the Children's Cancer Group and the Pediatric Oncology Group. Med Pediatr Oncol 1999, 33(4):362-371.

39. Paulino AC, Simon JH, Zhen W, Wen BC: Long-term effects in children treated with radiotherapy for head and neck rhabdomyosarcoma. Int J Radiat Oncol Biol Phys 2000, 48(5):1489-1495.

40. Cotter SE, Herrup DA, Friedmann A, Macdonald SM, Pieretti RV, Robinson G, Adams J, Tarbell NJ, Yock TI: Proton radiotherapy for pediatric bladder/ prostate rhabdomyosarcoma: clinical outcomes and dosimetry compared to intensity-modulated radiation therapy. Int J Radiat Oncol Biol Phys 2011, 81(5):1367-1373.

41. Rombi B, DeLaney TF, MacDonald SM, Huang MS, Ebb DH, Liebsch NJ, Raskin KA, Yeap BY, Marcus KJ, Tarbell NJ, Yock TI: Proton radiotherapy for pediatric Ewing's sarcoma: initial clinical outcomes. Int J Radiat Oncol Biol Phys 2012, 82(3):1142-1148.

42. Habrand JL, Schneider R, Alapetite C, Feuvret L, Petras S, Datchary J, Grill J, Noel G, Helfre S, Ferrand R, Bolle S, Sainte-Rose C: Proton therapy in pediatric skull base and cervical canal low-grade bone malignancies. Int J Radiat Oncol Biol Phys 2008, 71(3):672-675.

43. Rombi B, Ares C, Hug EB, Schneider R, Goitein G, Staab A, Albertini F, Bolsi A, Lomax AJ, Timmermann B: Spot-scanning proton radiation therapy for pediatric chordoma and chondrosarcoma: clinical outcome of 26 patients treated at Paul Scherrer Institute. Int J Radiat Oncol Biol Phys 2013, 86(3):578-584.

44. Krengli M, Hug EB, Adams JA, Smith AR, Tarbell NJ, Munzenrider JE: Proton radiation therapy for retinoblastoma: comparison of various intraocular tumor locations and beam arrangements. Int J Radiat Oncol Biol Phys 2005, 61(2):583-593

45. Sethi RV, Shih HA, Yeap BY, Mouw KW, Petersen R, Kim DY, Munzenrider JE, Grabowski E, Rodriguez-Galindo C, Yock TI, Tarbell NJ, Marcus KJ, Mukai S, Macdonald SM: Second nonocular tumors among survivors of retinoblastoma treated with contemporary photon and proton radiotherapy. Cancer 2014, 120(1):126-133.

46. Hattangadi JA, Rombi B, Yock TI, Broussard G, Friedmann AM, Huang M, Chen YL, Lu HM, Kooy H, MacDonald SM: Proton radiotherapy for high-risk pediatric neuroblastoma: early outcomes and dose comparison. Int $J$ Radiat Oncol Biol Phys 2012, 83(3):1015-1022.

47. Hill-Kayser C, Tochner Z, Both S, Lustig R, Reilly A, Balamuth N, Womer R, Maris J, Grupp S, Bagatell R: Proton versus photon radiation therapy for patients with high-risk neuroblastoma: the need for a customized approach. Pediatr Blood Cancer 2013, 60(10):1606-1611.

48. Fuji H, Schneider U, Ishida Y, Konno M, Yamashita H, Kase Y, Murayama S, Onoe T, Ogawa H, Harada H, Asakura H, Nishimura T: Assessment of organ dose reduction and secondary cancer risk associated with the use of proton beam therapy and intensity modulated radiation therapy in treatment of neuroblastomas. Radiat Oncol 2013, 8(1):255.

49. Hoppe BS, Flampouri S, Su Z, Morris CG, Latif N, Dang NH, Lynch J, Li Z, Mendenhall NP: Consolidative involved-node proton therapy for Stage IA-IIIB mediastinal Hodgkin lymphoma: preliminary dosimetric outcomes from a Phase II study. Int J Radiat Oncol Biol Phys 2012, 83(1):260-267.

50. Hoppe BS, Flampouri S, Su Z, Latif N, Dang NH, Lynch J, Joyce M, Sandler E, Li Z, Mendenhall NP: Effective dose reduction to cardiac structures using protons compared with 3DCRT and IMRT in mediastinal Hodgkin lymphoma. Int I Radiat Oncol Biol Phys 2012, 84(2):449-455.

51. Andolino DL, Hoene T, Xiao L, Buchsbaum J, Chang AL: Dosimetric comparison of involved-field three-dimensional conformal photon radiotherapy and breast-sparing proton therapy for the treatment of Hodgkin's lymphoma in female pediatric patients. Int J Radiat Oncol Biol Phys 2011, 81(4):e667-e671.

doi:10.1186/s13052-014-0074-6

Cite this article as: Rombi et al.: Proton radiotherapy for pediatric tumors: review of first clinical results. Italian Journal of Pediatrics 2014 40:74.

\section{Submit your next manuscript to BioMed Central and take full advantage of:}

- Convenient online submission

- Thorough peer review

- No space constraints or color figure charges

- Immediate publication on acceptance

- Inclusion in PubMed, CAS, Scopus and Google Scholar

- Research which is freely available for redistribution

Submit your manuscript at www.biomedcentral.com/submit
Ciomed Central 